\title{
Indentation of metals by a flat-ended cylindrical punch
}

\author{
B. Riccardi ${ }^{a}$, R. Montanari ${ }^{b}, *$ \\ ${ }^{a}$ Associazione EURATOM-ENEA, ENEA CR Frascati, PB 65, 00044 Frascati, Roma, Italy \\ ${ }^{\mathrm{b}}$ INFM-Dipartimento di Ingegneria Meccanica, Università di Roma-Tor Vergata, Via di Tor Vergata 110, 00133 Roma, Italy
}

Received 31 July 2003; received in revised form 8 April 2004

\begin{abstract}
The paper reviews the fundamentals of indentation theory for punches with cylindrical geometry, presents a deep-indentation finite element (FE) simulation and discusses an experimental technique for flat-ended cylindrical indentation. This technique is based on the use of cylindrical punches with diameters up to $1 \mathrm{~mm}$ and allows pressure-penetration curves to be drawn from which yield stress and elasticity modulus can be determined. Several materials have been tested including pure metals, steels and refractory alloys; yield stress has been determined and compared with literature values. By testing at different temperatures it was also possible to determine the ductile-to-brittle transition temperature (DBTT) for some alloys that show such phenomenon.
\end{abstract}

(C) 2004 Elsevier B.V. All rights reserved.

Keywords: Indentation; Mechanical tests; Ductile-to-brittle transition temperature; Finite element analysis

\section{Introduction}

Since more than a century, the indentation test has been one of the most common techniques for the mechanical characterization of materials. The test output is usually the indentation depth or the size of the imprint, which are related to a hardness index number. Different hardness tests have been standardized, depending on indenter geometry and indentation parameters. Indentation techniques have been also used to study time and temperature dependent properties of metals such as creep and load relaxation [1,2], or to estimate the residual stress field and fracture toughness of brittle materials $[3,4]$. Recently, low-load indentation techniques have been developed for testing metallic and ceramic thin films or coatings [5]. When the indentation test is operated on a micro- or nano-scale, factors such as micro-segregation of chemical elements, surface roughness, residual strain pattern resulting from manufacturing processes can contribute to produce a large scattering of results, so that several tests and statistical treatment of data are necessary [6].

Abbreviations: FE, finite element; FEM, finite element modelling; FIMEC, flat top indenter for mechanical characterization; LVDT, linear variable differential transformer; DBTT, ductile-to-brittle transition temperature

* Corresponding author. Tel.: +3906 72597182; fax: +39062021351.

E-mail address: roberto.montanari@uniroma2.it (R. Montanari).
Theoretical fundamentals of the indentation problem, that are elastic and (within certain limits) plastic, have been formulated for different indenter geometries. Different models have also been set up to predict indentation hardness and to estimate uniaxial mechanical properties for all the geometries (cone, wedge or pyramid, sphere, flat-ended cylinder). Recently, a comprehensive model to describe the behaviour of a wide variety of materials tested with different indenter geometries has been developed [7]. This model expresses the analytical equations of hardness as a function of the material uniaxial properties. Finally, the indentation problem has been successfully approached by the finite element modelling (FEM) of indentations performed by a fully rigid punch on an elastic plastic half-space, both with and without friction $[8,9]$.

Indentation with sharp punches has been widely used for material characterisation, but also applications of flat-ended cylindrical indenters can be found in literature [10-14]. They concern investigations on mechanical properties of materials and measurements of the elastic modulus of thermal barrier coatings [15].

The indentation with sharp or spherical indenters involves that the contact surface area increases as the load increases; conversely, during the indentation with cylindrical punches, the contact area remains constant; thus, the contact pressure linearly increases with indentation load. In this paper, a flat-ended cylindrical indentation technique (FIMEC), 
Table 1

Comparison between yield stress $\sigma_{\mathrm{y}}$ obtained by tensile tests and $p_{\mathrm{y}} / 3$ by FIMEC tests

\begin{tabular}{|c|c|c|c|}
\hline \multirow[t]{2}{*}{ Material } & \multirow{2}{*}{$\begin{array}{l}\text { Tensile test, } \\
\sigma_{\mathrm{y}}(\mathrm{MPa})\end{array}$} & \multicolumn{2}{|l|}{ FIMEC test } \\
\hline & & $p_{\mathrm{y}} / 3(\mathrm{MPa})$ & Deviation \\
\hline $\mathrm{Al}$ & 60 & 55 & 0.08 \\
\hline $\mathrm{Cu}$ & 190 & 185 & 0.03 \\
\hline $\mathrm{Zn}$ & 55 & 55 & 0 \\
\hline Mo & 540 & 560 & -0.04 \\
\hline Composite $\mathrm{Al}-7 \% \mathrm{Si}+\mathrm{SiCp}$ & 280 & 275 & 0.02 \\
\hline A986 (Al alloy) & 35 & 32 & 0.09 \\
\hline Glidcop $\left(\mathrm{Cu}-0.48 \mathrm{Al}_{2} \mathrm{O}_{3}\right)$ & 483 & 460 & 0.05 \\
\hline $\mathrm{Cu}-5 \mathrm{Zn}-7 \% \mathrm{Zr}$ & 306 & 310 & -0.01 \\
\hline $\mathrm{Fe}-40 \% \mathrm{Al}+1 \% \mathrm{Y}_{2} \mathrm{O}$ & 922 & 900 & 0.02 \\
\hline $\mathrm{W}+1 \% \mathrm{La}_{2} \mathrm{O}_{3}$ & 765 & 710 & 0.07 \\
\hline TZM (Mo alloy) & 960 & 910 & 0.05 \\
\hline AISI 1040 steel & 450 & 425 & 0.06 \\
\hline MANET II steel & 640 & 640 & 0 \\
\hline BATMAN-1951 steel & 510 & 500 & 0.02 \\
\hline F82H steel & 520 & 500 & 0.04 \\
\hline AISI $316 \mathrm{~L}$ steel & 310 & 330 & -0.06 \\
\hline
\end{tabular}

The deviation $\Delta$ is $\left(\sigma_{\mathrm{y}}-p_{\mathrm{y}} / 3\right) / \sigma_{\mathrm{y}}$.

which has been recently developed by us, is presented and discussed [14,16-18]. This technique employs cylindrical indenters with diameters ranging from 0.5 to $1 \mathrm{~mm}$ and gives pressure-penetration curves from which yield stress and elasticity modulus can be determined. Experiments have been performed on several metals (Table 1). From tests performed at different temperatures it is also possible to have an indication about the ductile-to-brittle transition temperature (DBTT) for those metals which show such phenomenon [19].

The specific characteristics of FIMEC are:

(i) the high simplicity of the apparatus and data treatment;

(ii) the possibility to get information about the local material properties on a scale large enough to include many grains; thus, data represent bulk characteristics and are not influenced by those factors which dramatically affect micro- and nano-indentation tests;

(iii) the large versatility in industrial applications such as the control of welding quality [20,21], the on-line monitoring of forging or extrusion processes, etc.

In this paper, also the fundamentals of the theory of indentation by a cylindrical punch are reviewed and a numerical finite element (FE) simulation of a deep indentation by a $1 \mathrm{~mm}$ diameter punch is presented.

\section{Indentation theory}

\subsection{Elastic models}

The problem of the contact of two elastic (spherical) bodies was originally developed by Hertz [22], but the approach to the problem of an elastic half-space subjected to a pres-

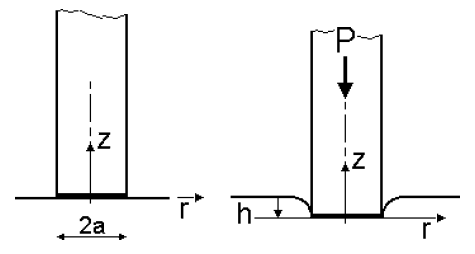

Fig. 1. Flat-ended indentation scheme.

sure acting on a closed surface is due to Cerruti [23] and Boussinesq [24], who used the potential theory method, although the solution found cannot be used for problems of a practical interest. Love [25] managed to find a solution for conical and cylindrical indenters. Finally, Sneddon [26] derived the load-displacement relations for an arbitrary shaped axisymmetric punch. The results of the Sneddon solution for the indentation without friction of an elastic half-space by a flat-ended cylindrical punch, can be summarised as follows (see Fig. 1). The contact area is assumed to be circular and equal to the indenter tip area (radius $a$ ). The boundary conditions for the formulation $(z=0$ for the local system $r-z$ ) are the following:

$$
\begin{array}{lc}
\sigma_{z}(r, 0)=0, & r>a \\
\tau_{r z}(r, 0)=0, & 0 \leq r \leq a \\
u_{z}(r, 0)=h, & 0 \leq r \leq a
\end{array}
$$

The first condition imposes that the free surface outside the contact region has no normal stress $\left(\sigma_{z}\right)$ acting on it; the second one assures the absence of any friction for the contact region between the indenter and the half space; the third one forces the displacement in $z$ direction $\left(u_{z}\right)$ to be consistent with the flat facet of the punch. Since the punch has a sharp edge $\sigma_{z} \rightarrow \infty$ when $r=a$. This condition [27] determines a very localized plasticity on the circular edge that does not invalidate the effectiveness of the elastic relationships found, in particular, the load-displacement and the contact pressure equations.

Following a penetration $h$, the mean contact pressure $p_{\mathrm{m}}$ is given by the equation:

$p_{\mathrm{m}}=\frac{2 E h}{\pi a\left(1-v^{2}\right)}$

which shows that the relationship between $p_{\mathrm{m}}$ and $h$ is linear due to the constant contact area.

The distribution of pressure under the punch $\sigma_{z}(r \leq a, z$ $=0$ ) is given by the equation:

$\sigma_{z}(r, 0)=-\frac{p_{\mathrm{m}}}{2 \sqrt{1-r^{2} / a^{2}}}$

and the shape of the deformed boundary $u_{z}(r>a, z=0)$ by:

$u_{z}(r, 0)=\frac{2 h}{\pi} \arcsin \left(\frac{a}{r}\right)$

The equations governing the stress field in the elastic half-space coming from the Sneddon solution [26,28-30] 
are given in the $(r, \theta, z)$ cylindrical coordinate system by the following equations:

$$
\begin{aligned}
\sigma_{r}= & -\frac{1}{2} p_{\mathrm{m}}\left\{\left[-z \frac{\mathrm{d}^{2}}{\mathrm{~d} z^{2}}-\frac{\mathrm{d}}{\mathrm{d} z}\right] \arctan \left[\frac{1+K \sin \Phi}{z+K \cos \Phi}\right]\right. \\
& \left.-\frac{1}{r^{2}}\left[(1-2 v)+z \frac{\mathrm{d}}{\mathrm{d} z}\right](1-K \sin \Phi)\right\} \\
\sigma_{\vartheta}= & p_{\mathrm{m}}(1+v) \frac{\mathrm{d}}{\mathrm{d} z} \arctan \left[\frac{1+K \sin \Phi}{z+K \cos \Phi}\right]-\sigma_{r}-\sigma_{z} \\
\sigma_{z}= & -\frac{1}{2} p_{\mathrm{m}}\left[z \frac{\mathrm{d}^{2}}{\mathrm{~d} z^{2}}-\frac{\mathrm{d}}{\mathrm{d} z}\right] \arctan \left[\frac{1+K \sin \Phi}{z+K \cos \Phi}\right] \\
\tau_{r z}= & -\frac{1}{2} p_{\mathrm{m}} \frac{z}{r} \frac{\mathrm{d}^{2}}{\mathrm{~d} z^{2}}(1-K \sin \Phi)
\end{aligned}
$$

with the assumption $a=1$ for simplicity and with

$$
K^{4}=\left(r^{2}+z^{2}-1\right)+4 z^{2} \text { and } \tan (2 \Phi)=\frac{2 z}{r^{2}+z^{2}-1}
$$

\subsection{Elastic-plastic model}

An indentation model which also includes plasticity is much more complex. The complexity is due to the fact that the constitutive equations are not linear and include some material parameters able to describe the plastic behaviour, such as the yield stress and the work-hardening coefficient.

Two models have been developed to describe the material stress-strain field, following an indentation performed by means of punches of different shapes: namely, the spherical cavity [31] and the slip-line model [32]. The first one was suggested by the consideration that for some indenter shapes, such as the spherical and blunt conical ones, the plastic zone shows spherical symmetry (hemispherical), thus the problem can be approached in analogy with the elastic-plastic deformation near a cavity subjected to internal pressure. Unfortunately, this model is too rough to be applied to a cylindrical indentation because the plastic region shape varies with penetration depth and becomes roughly spherical only at elevated depth values [7,33].

The slip-line approach is suitable for the modelling of material indentation that can be described by means of a rigid plastic model (i.e. no hardening), where the plastic deformation is much higher than the elastic one, so that only plastic deformation can be considered. During indentation, and differently from the spherical cavity model which involves a compressive action, the material is supposed to flow at a constant shear or normal stress under a punch "cutting action", which determines a new surface on the indented specimen. In these conditions, the stress in the flow region can be described by means of the slip-line field theory. This approach allows a grid of curvilinear lines to be determined: on this grid, the plastic field can be described by means of a constant simple shear stress and a variable hydrostatic stress. This approach has been successfully applied to solve plane strain indentation (e.g. that produced by a wedge punch) but cannot be applied to solve the general axisymmetric problem.

Shield [34] showed that the axisymmetric plastic flow of a rigid-plastic material can be described by a slip-line field if the material follows the Tresca plasticity criterion, and assuming the circumferential stress equal to one of the principal stress in the meridional plane (Haar-von Karman hypothesis). Under these hypotheses, Shield determined the plastic stress field for the indentation of a semi-infinite solid by a smooth flat-ended cylindrical punch. He also extended the plastic stress field outside the punch contact region and estimated the value of the average contact pressure $p_{\mathrm{m}}=$ $5.69 k$ ( $k$ being the shear strength of the material). In the same conditions, the maximum contact pressure (close to punch edge) was found to be $p_{\max }=7.2 k$ and the radial extension of the plastic area is $1.58 a$.

Afterwards, Eason and Shield [35] studied the indentation of a semi-infinite solid by a rough punch evaluating also the radial extension and depth of plastic zone. As already noticed, due to the discontinuity of contact pressure under the flat-ended cylindrical punch, the plasticity starts at punch contact edge; then it progressively extends with increasing penetration. The result of Shield-Eason approach is that the plasticity extension reaches the indenter-specimen axis when the mean contact pressure is about six times the shear strength; thus, as consequence of the Tresca plasticity criterion, when the contact pressure is about three times the tensile yield stress.

\section{Numerical simulation}

As already noticed, the indentation of an elastic-perfectlyplastic material is a problem difficult to be solved analytically and the major part of this difficulty is due to the unknown shape and extension of the plastic field. In certain hypotheses, a solution can be found, but in any case only for small plastic strains. Therefore, it is not possible to describe any deep-indentation process [32].

These difficulties can be avoided by means of a finite element analysis when a refined mesh is used to model the continuum half-space, a suitable material formulation is provided and finally the contact is properly modelled. In this paper, two approaches are presented: the first one concerns a basic simulation of the indentation of an elastic-plastic material with and without strain hardening and friction; the second one concerns the simulation of the experimental indentation carried out on a martensitic steel (included in the experimental Section 4).

\subsection{The model}

All the calculations (basic analysis and experimental simulation) have been performed by means of an axisymmetric model and a geometrical non linear analysis has been carried out. This model consists of a perfectly rigid flat-ended 


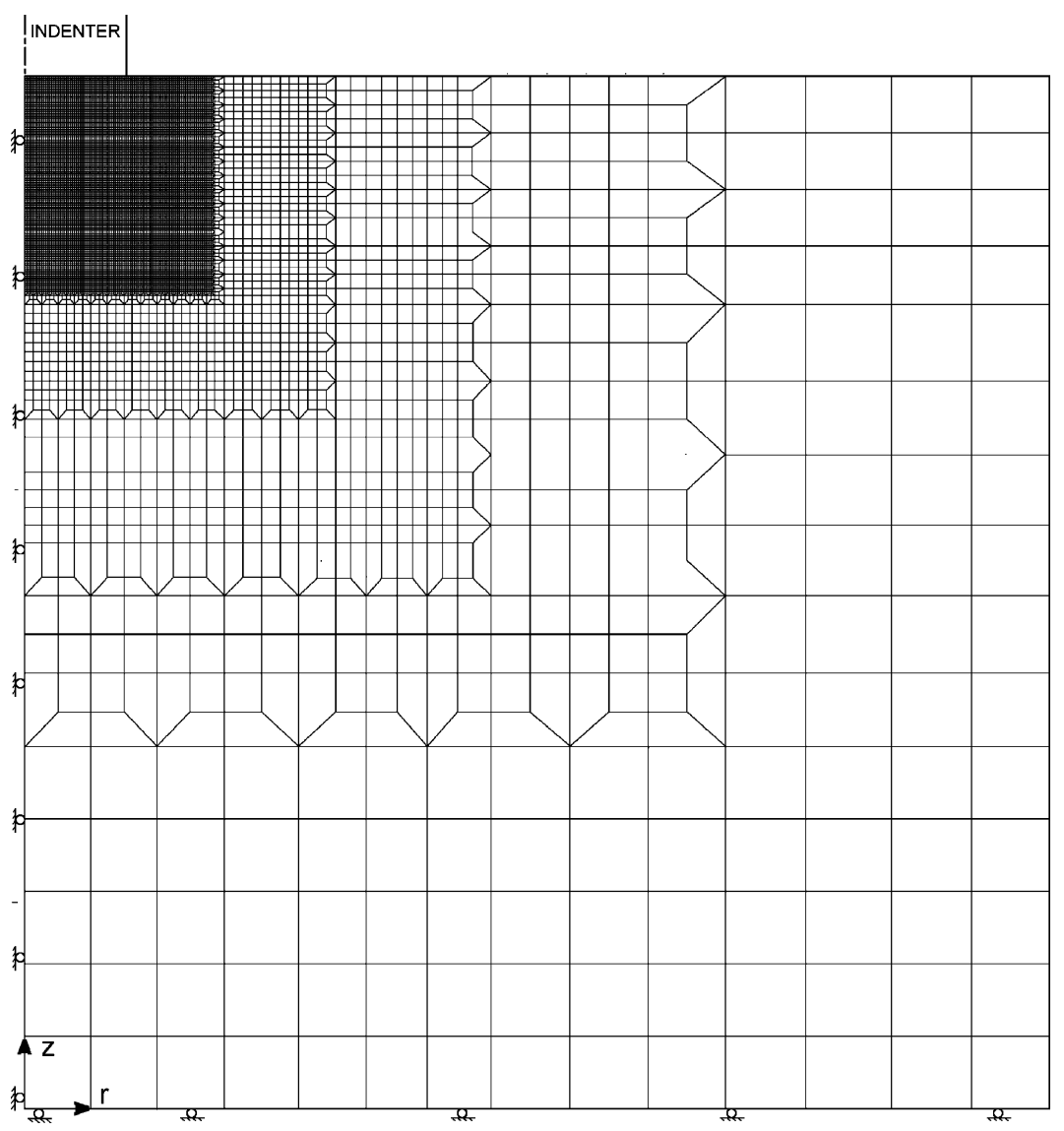

Fig. 2. FE model.

cylindrical punch (radius $0.5 \mathrm{~mm}$ ) which penetrates a specimen (radius $5 \mathrm{~mm}$, thickness $5 \mathrm{~mm}$ ). Experimental measurements by modulated optical reflectivity [17] and a sensitivity FE analysis showed that these dimensions are sufficient to consider the specimen as a semi-infinite solid. The analysis has been carried out by means of the general purpose code Abaqus [36,37]. The indenter has been modelled by means of a rigid surface while the specimen by means of a mesh of 7200 four nodes reduced integration axisymmetric elements (Fig. 2). Roller boundary conditions have been imposed along the symmetry axis and at the bottom of the specimen (frictionless rigid boundary). The mesh size chosen is the result of an optimisation carried out in order to describe the deformation process close to the indenter edge; a $10 \mu \mathrm{m}$ size element was found to reach a good compromise in terms of computer time and accuracy of results. The simulation has been performed by imposing 600 penetration steps $(0.1 \mu \mathrm{m}$ each). For the basic calculations, the material was considered homogeneous, isotropic and fully described by means of the elastic modulus $E$, the Poisson coefficient $v$ and the yield stress $\sigma_{\mathrm{y}}$ whereas the viscosity has not been considered. A parametric study of the influence of the friction between indenter and sample, and of the material hardening rate was also carried out.

\subsection{Results}

FE calculations have been performed first by assuming the friction coefficient $f$ as a parameter $(f=0,0.1,0.5)$ and an elastic-perfectly-plastic material behaviour with no hardening $\left(E=2.1 \times 10^{5} \mathrm{MPa}, v=0.3, \sigma_{\mathrm{y}}=500 \mathrm{MPa}\right)$. The indentation curves, as proposed by Yu [7], have been normalized by plotting $p_{\mathrm{m}} / \sigma_{\mathrm{y}}$ versus $2 E /\left[\pi\left(1-v^{2}\right) \sigma_{\mathrm{y}}\right] h / a$ and are shown in Fig. 3. Each curve has a linear initial stage, whose slope can be derived from Eq. (2); the linear stage is followed by a curved part and finally, at high penetration values, by an almost constant slope stage. The results of this simulation point out that friction coefficient effect is practically negligible at least up to the penetration values investigated here.

In the case of frictionless contact, the trend of the curve in Fig. 3 is given with good approximation by the equation:

$\xi=c_{1} \frac{\chi}{1+\chi^{c}}$

where $c_{1}=2.55, c=0.93, \xi=p_{\mathrm{m}} / \sigma_{\mathrm{y}}$ and $\chi=2 E /[\pi(1-$ $\left.\left.v^{2}\right) \sigma_{\mathrm{y}}\right] h / a$. The qualitative stress-strain field under the indenter is shown in Fig. 4(a-d) for a penetration parameter $h / a=0.1$ (corresponding to $\chi=27.9$, i.e. in the constant slope stage of the load-penetration curve). The hydrostatic 


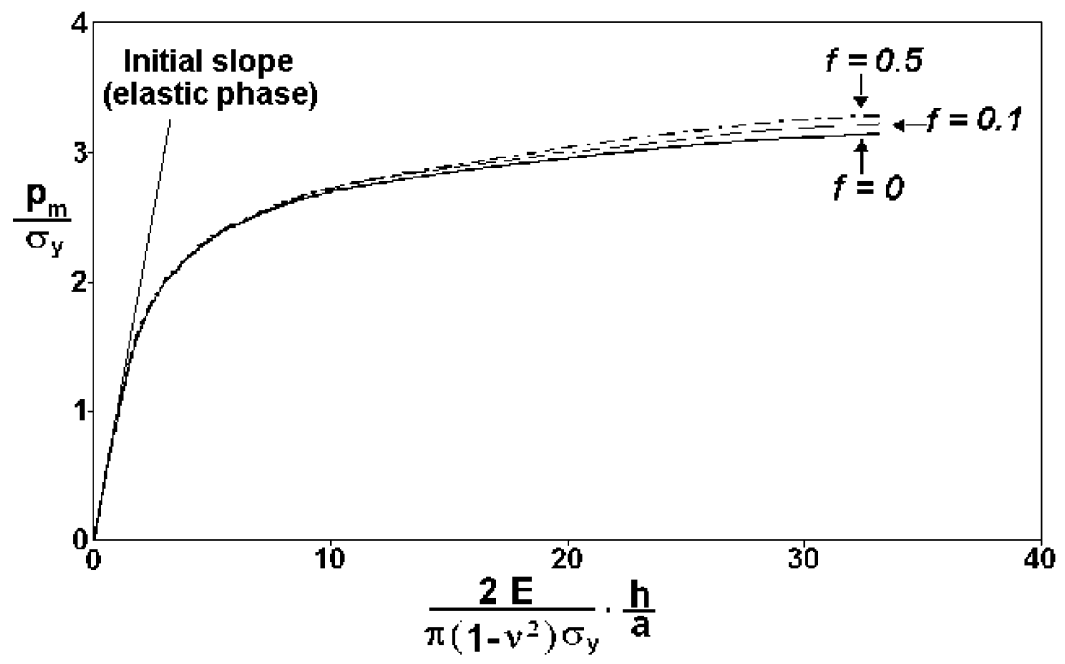

Fig. 3. FE normalized indentation curves calculated with three different friction coefficients.
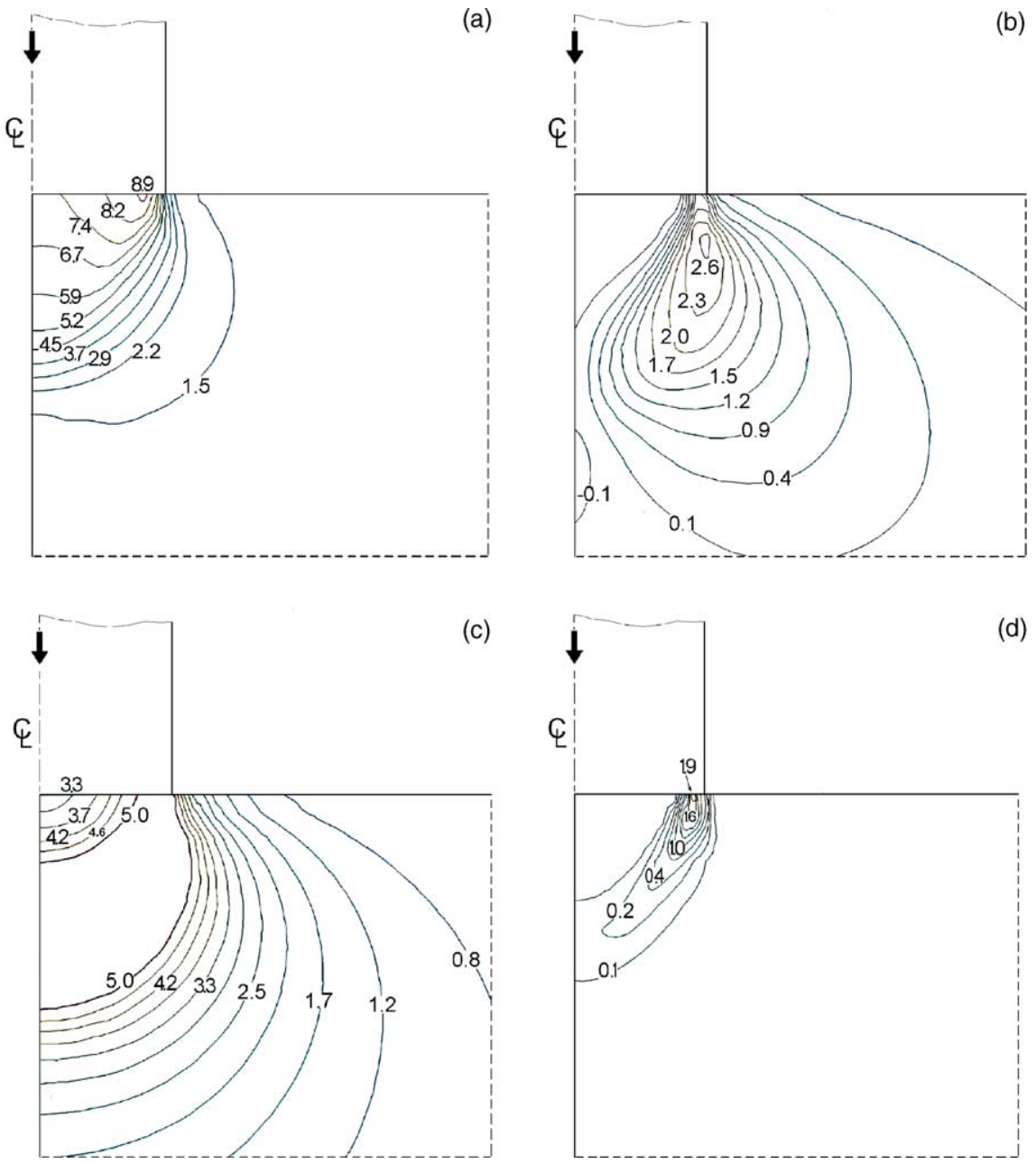

Fig. 4. Elastic-perfectly-plastic, frictionless FE analysis contour at $h / a=0.1$ : (a) hydrostatic pressure $(\times 100 \mathrm{MPa})$; (b) shear stress $(\times 100 \mathrm{MPa})$; (c) von Mises equivalent stress ( $\times 100 \mathrm{MPa})$; (d) von Mises equivalent plastic strain. 


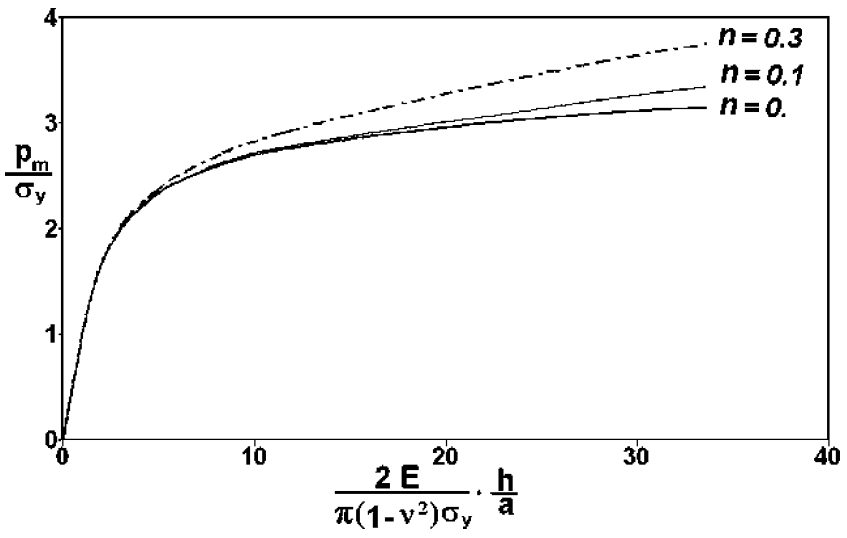

Fig. 5. FE normalized indentation curves calculated with three different hardening rates.

pressure stress distribution inside the material shows that the maximum value occurs at $r=0.8 a$. It would have been useful to compare the calculated strain with some experimentally measured values. Unfortunately, only measurements of strain distribution around Vickers indentations are reported in literature [38]. Therefore, this will be the next step of our activity.

Simulations have been also carried out to estimate the effect of the hardening rate in the case of frictionless contact if the material properties remain unchanged. Fig. 5 shows the indentation curves calculated with different hardening rates $n(0,0.1$ and 0.3$)$. The effect of the hardening rate appears to be modest in the initial part of the curve, but is remarkable on the final slope.

\section{Experimental}

\subsection{The apparatus}

The FIMEC indentation procedure is based on the penetration of a flat punch at constant rate. Usually, a punch with diameter $=1 \mathrm{~mm}$ and axial length $=1.5 \mathrm{~mm}$ is employed in the tests, however, punches of reduced size (diameters up to $0.5 \mathrm{~mm}$ ) can also be used, depending on the extension of the zone to examine.

The experimental set-up is sketched in Fig. 6, which also shows a picture of the apparatus. A massive frame, dimensioned to have a total elastic deformation of about $1 \mu \mathrm{m}$ for the maximum applied load $(10 \mathrm{kN})$, hosts all the components of the apparatus: the linear actuator which drives the indenter advancement, the load cell, the displacement measuring system (LVDT) and optionally also a thermostatic container. The linear actuator is an electro-mechanical drive equipped with a step motor. The motor rotation is transmitted to a ball screw by a precision reduction gear; the ball screw converts the rotation at the gear output to translation, guided by means of a pre-loaded ballspline. The flat punch indenter is made of tungsten carbide which guarantees low deformability and high strength. The indenter is mounted at the end of a rod and the advancement speed can be varied in the range from $1 \times 10^{-4}$ to $2 \times 10^{-2} \mathrm{~mm} / \mathrm{s}$. The motor con-
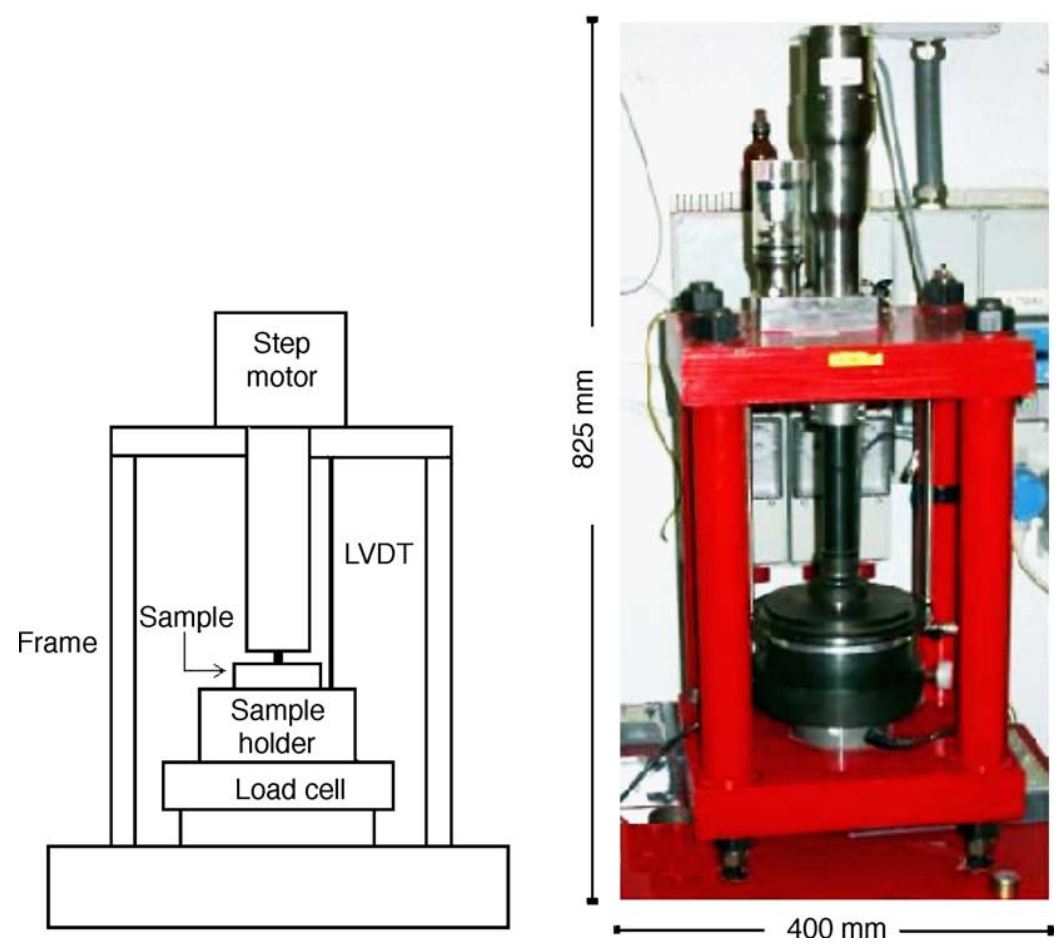

Fig. 6. FIMEC scheme and apparatus. 
trol unit is connected to the LVDT and a feedback system provides constant speed in the full range of the load applied. The LVDT system measures the displacement between the sample holder and the indenter with a resolution of $1 \mu \mathrm{m}$ and the load cell, located under the sample holder, measures the applied load with a resolution of $1 \mathrm{~N}$. Tests at low and high temperatures, in the range from -180 to $200^{\circ} \mathrm{C}$, can be performed by using a thermostatic device.

To control the position of each indentation on the sample, and to optimise the number of indentations, the sample holder is mounted on a plate that can rotate and translate. A distance of $5 \mathrm{~mm}$ between the centres of two adjacent indentations has been determined to be the minimum distance to avoid overlapping of the strain fields of neighbouring imprints, which can affect the pressure-penetration curves [17]. So, for example, it is possible to perform up to 18 indentations on a disk-shaped sample with diameter $25 \mathrm{~mm}$ and thickness $5 \mathrm{~mm}$.

The entire indentation process is controlled by a software specifically developed for the instrument.

For each test temperature and tested material, at least five indentations are performed and generally the results show a very good reproducibility. All the data presented in this paper are mean values.

\subsection{Experimental results}

The typical trend of an experimental indentation curve with the different stages is evidenced in Fig. 7 for F82H steel (a 9\% $\mathrm{Cr}$ martensitic steel stabilized by $2 \% \mathrm{~W}$ ). A punch imprint and a micrographic section are shown in Fig. 8(a and $b$ ).

The curves of all the tested materials show a trend characterized by an initial linear elastic stage up to a pressure load $p_{\mathrm{L}}$. Below $p_{\mathrm{L}}$ the curve is fully reversible and no permanent deformation occurs on the sample. The linear stage is followed by three plastic stages:

- the first one is almost linear and ends at a pressure $p_{\mathrm{y}}$ : the imprint shows permanent sharp edges;

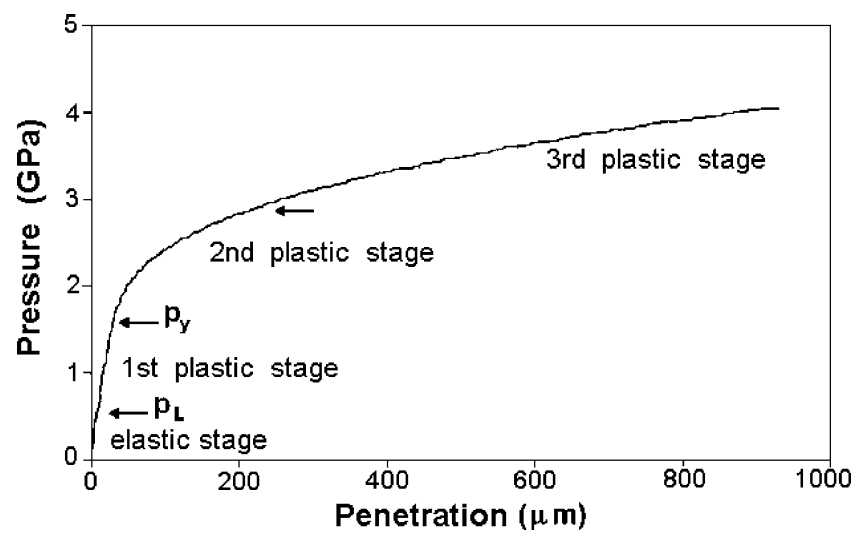

Fig. 7. Experimental pressure-penetration curve for F82H steel.

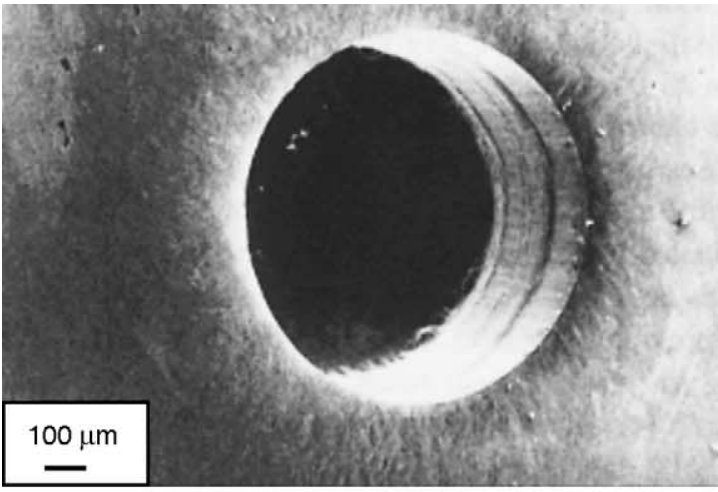

(a)

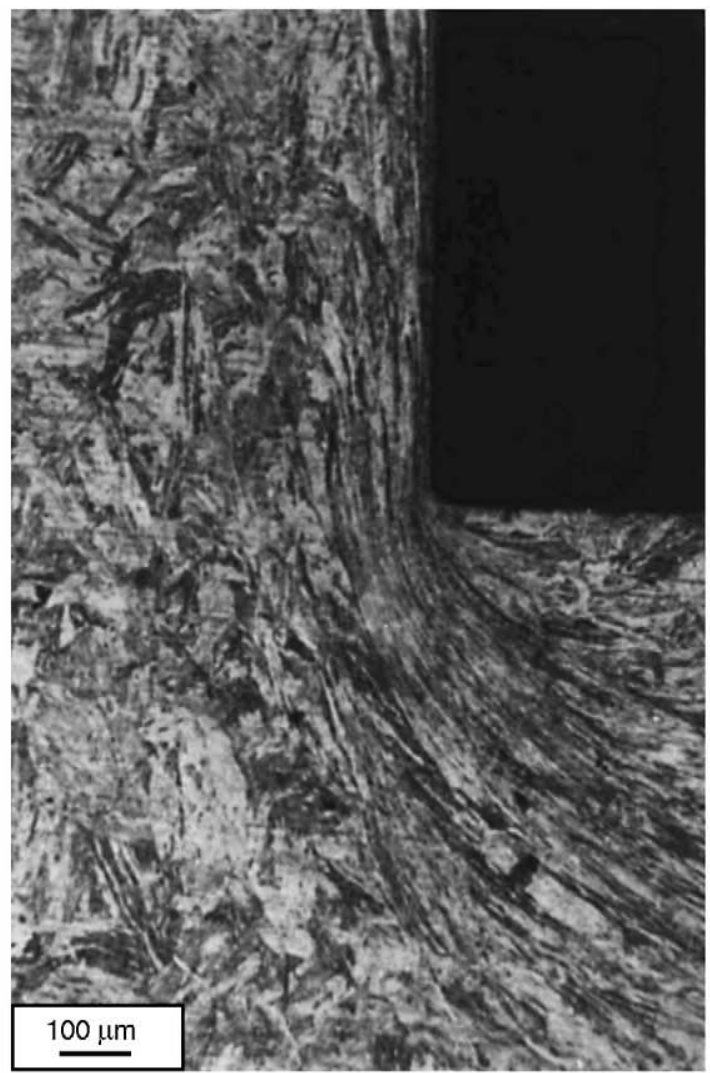

(b)

Fig. 8. Punch imprint (a) and its micrographic section (b).

- the second one occurs for $p>p_{\mathrm{y}}$ and is characterized by a sudden slope decrease. During this stage the material starts to protrude around the imprint;

- the third stage shows a trend with an almost constant slope.

For some materials the third stage evidences a saturation pressure load $p_{\mathrm{s}}$ which is strongly influenced by the penetration rate, while the $p_{\mathrm{y}}$ load is scarcely affected by this parameter [17]. This last result holds for all the investigated materials and is of great importance to correlate indentation and tensile test results. When the indentation tests are carried out with a penetration rate of $0.1 \mathrm{~mm} / \mathrm{min}$ or lower, it is possible to compare directly the indentation results with 


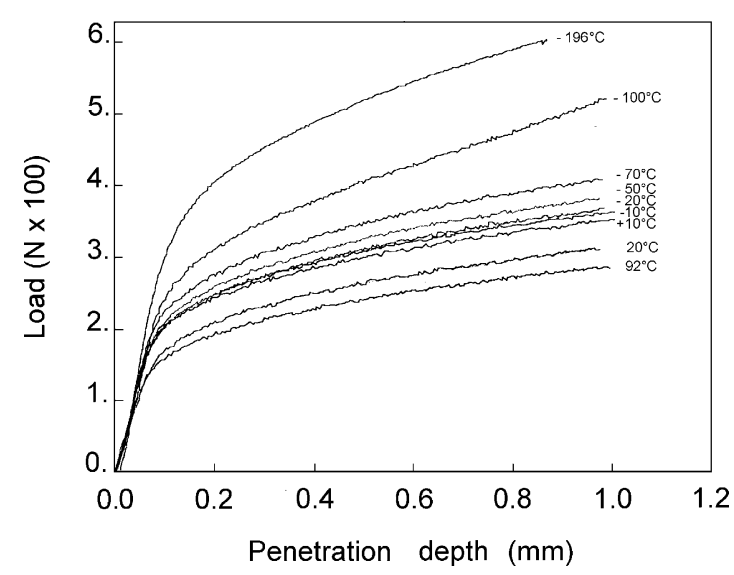

Fig. 9. Experimental load-penetration curves at different temperatures for F82H steel.

those of tensile tests with a strain rate of $10^{-3} \mathrm{~s}^{-1}$; in these conditions it has been observed that the yield stress $\sigma_{\mathrm{y}}$ is approximately equal to $p_{\mathrm{y}} / 3$. This result is similar to the finding of Yu et al. [13]. They found that, when plastic flow occurs during a cylindrical indentation, the ratio between indentation pressure and compression (yield) stress is not widely different from value 3 . Moreover, according to the results of Yu et al. [13], the pressure-penetration curves obtained in this work by testing the same material with indenters of different diameters $(1.0,0.8$ and $0.7 \mathrm{~mm})$ show an almost perfect overlapping [18].

Several metals have been tested and Table 1 lists for some of them the yield stress $\left(\sigma_{\mathrm{y}}\right)$ values from standard tensile tests, the $\mathrm{p}_{\mathrm{y}} / 3$ values coming from indentation curves and the relative difference of these two values $\Delta=\left(\sigma_{\mathrm{y}}-p_{\mathrm{y}} / 3\right) / \sigma_{\mathrm{y}}$. This difference does not exceed $9 \%$, a value similar to the scattering of data (about 7\%) in tensile tests on the same material [39].
Table 2

Ductile to brittle transition temperature (DBTT) of three martensitic steels obtained by different methods

\begin{tabular}{llll}
\hline Material & $\begin{array}{l}\text { DBTT (ISO-V) } \\
\left({ }^{\circ} \mathrm{C}\right)\end{array}$ & $\begin{array}{l}\text { DBTT (KLST) } \\
\left({ }^{\circ} \mathrm{C}\right)\end{array}$ & $\begin{array}{l}\mathrm{DBTT}_{\text {FIMEC }} \\
\left({ }^{\circ} \mathrm{C}\right)\end{array}$ \\
\hline MANET & -35 & - & -50 \\
BATMAN & -15 & -75 & -85 \\
F82H & -45 & -80 & -79 \\
\hline
\end{tabular}

Fig. 9 shows the $p-h$ curves for $\mathrm{F} 82 \mathrm{H}$ steel at different temperatures. The slope of the third plastic stage $(\Delta p / \Delta h)$ depends on the test temperatures. In particular, the curves obtained at lower temperature exhibit higher $\Delta p / \Delta h$ slopes and slightly higher $p_{\mathrm{y}}$ values. Therefore, $\Delta p / \Delta h$ has been used to correlate the results of FIMEC and Charpy tests to have an estimation of the ductile to brittle transition temperature [17]. Fig. 10 shows the mean value of $\Delta p / \Delta h$ in the third plastic stage versus the test temperature for $\mathrm{F} 82 \mathrm{H}$ steel. The curve has been plotted using a reversed ordinate scale to show how the trend is similar to that of a resilience curve. The DBTT has been determined as the abscissa corresponding to the average value $\mathrm{M}$ between the upper (U) and the lower (L) values in the plot. The DBTT of several steels have been determined by FIMEC [19]. Table 2 compares the DBTT values of three martensitic steels obtained from FIMEC and Charpy tests performed by using standard ISO-V and sub-sized $(3 \mathrm{~mm} \times 4 \mathrm{~mm} \times 27 \mathrm{~mm})$ KLST specimens.

The DBTT values from FIMEC are very close to those obtained by Charpy tests with KLST specimens while larger differences are observed with data from tests employing ISO-V probes.

One of the main interest of FIMEC test is a direct estimation of the elastic modulus $E$ because the cylindrical indentation loading/unloading curve is linear provided that

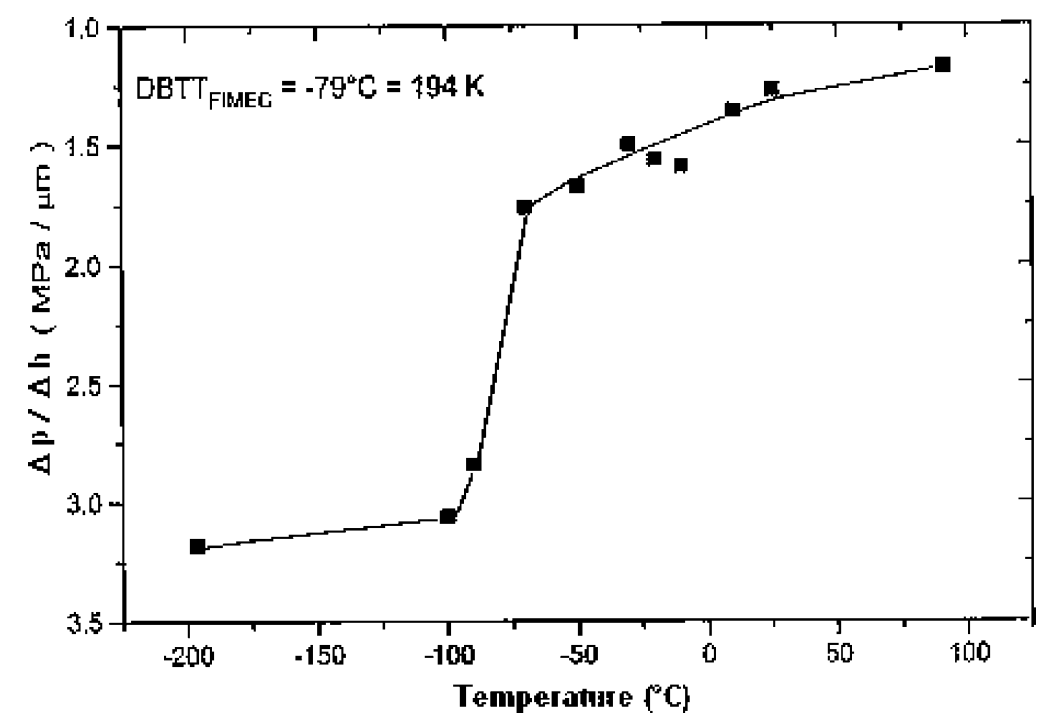

Fig. 10. Determination of DBTT for F82H steel. 
the sample deformation is only elastic [15]. The FIMEC indentation allows this property to be determined from the unloading part of the pressure-penetration curve by means of the elastic relationship (2). In principle, also the loading part could serve the scope but possible punch-sample imperfect contact and back-lash in the movement transmission introduce some uncertainties in the measurement, so it is convenient to estimate $\mathrm{E}$ from the unloading part of the curve. As observed also by Oliver et al. [40], in a deep indentation of metals, most of the indenter displacement is accomodated by sample plastic deformation and only a small amount related to the elastic deformation. The unloading part of the flat punch indentation (in ideal experimental conditions) is expected to be linear back to zero load. In our case, due to problems of contact mismatches (the recovered surface is not flat or parallel thus the indenter experiences a receding area of contact as it disconnects from the sample) and possible friction between punch and specimen, only the initial part of the unloading curve is linear. Therefore, this initial part has been used for the estimation of the elastic modulus. The elastic modulus is estimated by means of the slope of the initial part of the unloading curve (contact stiffness $S=\mathrm{d} P / \mathrm{d} h)$ by using the equation [40]:

$S=\frac{\mathrm{d} P}{\mathrm{~d} h}=\frac{2}{\sqrt{\pi}} E_{\mathrm{eq}} \sqrt{A}$

$E_{\text {eq }}$ is the equivalent modulus defined by $[22,41]$ :

$\frac{1}{E_{\mathrm{eq}}}=\frac{1-v^{2}}{E}+\frac{1-v_{\mathrm{i}}^{2}}{E_{\mathrm{i}}}$

where $E_{\mathrm{i}}, E$ and $v_{\mathrm{i}}, v$ are the Young's modulus and Poisson's coefficient of indenter and sample.

The flat top cylindrical indentation test has the big advantage (with respect those performed by means of sharp indenters) that the contact area $A$ is independent on indentation depth. Fig. 11 shows for F82H steel a typical loading-unloading curve. Several tests performed showed that the slope of the initial part of unloading curves is substantially independent from the penetration depth. For example, from the unloading part of the curve reported in Fig. 11 a value $E_{\mathrm{eq}}=162 \mathrm{GPa}$ was determined by means of Eq. (11). By assuming $E_{\mathrm{i}}=668.35 \mathrm{GPa}, v_{\mathrm{i}}=0.24$ [42] and $v=$ 0.33 , the elastic modulus E calculated from Eq. (12) was $210.5 \mathrm{GPa}$. This value differs from that obtained by tensile tests (205 GPa) by only $3 \%$; it can therefore be considered to be a good estimation.

\subsection{Numerical simulation}

A finite element analysis of the indentation process was carried out by means of the same geometric model used in the ideal elastic-plastic case. The real indenter has been modelled as perfectly rigid and this assumption can be considered sufficiently approximated by taking for the material the equivalent elastic modulus $E_{\text {eq }}$ as defined by Eq. (12).

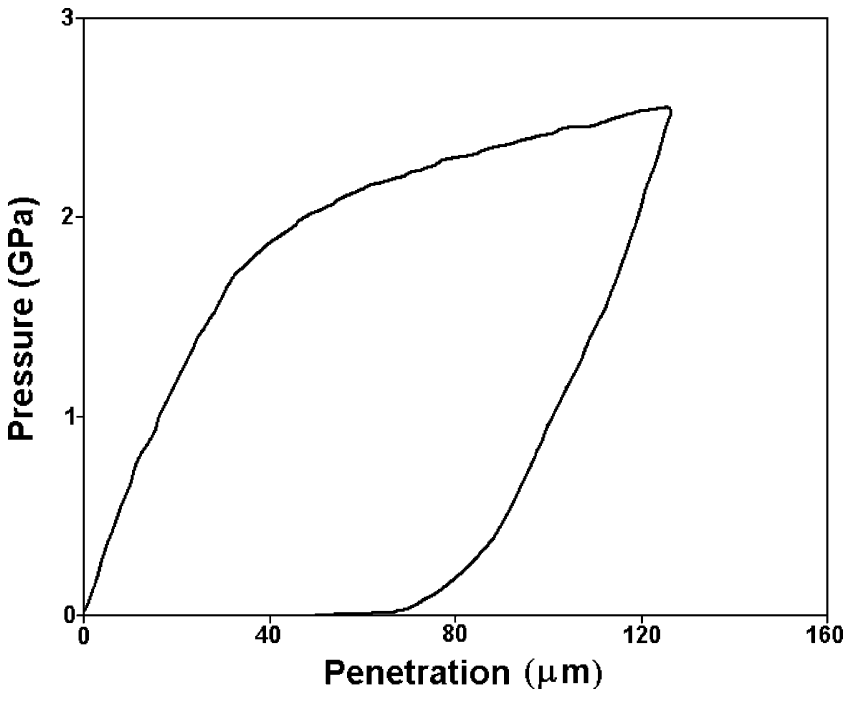

Fig. 11. Experimental loading-unloading curve for F82H steel.

A study of deep penetration (up to $1 \mathrm{~mm}$ ) was carried out by means of a geometric non linear-adaptive mesh-dynamic analysis [37]. The adaptive mesh option was used in order to deal with the severe distortion of the FE mesh used for the model; in this way it was possible to re-arrange locally the model mesh when the distortion of each element exceeds a pre-defined value. This is a very heavy procedure that Abaqus Explicit code allows to be both done and controlled automatically. This analysis, even if performed by means a dynamic code, is representative of the penetration process because the used parameters allowed to get a quasi static response. Conversely, the results of the simulation, which did not account for the material viscosity, only led to a rough estimation of strain amplitude and pattern. The analysis also gave a preliminary indication of how it is possible to reproduce the imprint profile.

The analysis has been focussed on the F82H indentation at room temperature; the plastic part of the stress strain curve was modelled by means of the Holloman's relationship $\sigma$ $=k \varepsilon^{n}$ where $k=813.63 \mathrm{MPa}$ and the average work hardening coefficient $n=0.07168$ [18]. In the plastic range, the material was assumed to follow the von Mises plasticity criterion.

The von Mises equivalent plastic strain contours are shown in Fig. 12 after a penetration of $1 \mathrm{~mm}$.

The main finding of the F82H steel FEM indentation analysis, obtained by plotting several plastic strain contours and deformed plots at increasing penetration depth, was that a consistent extension of plastic region under the punch and the occurrence of plastic flow and protrusion (pile up) can be observed at a pressure value $\left(p_{\mathrm{y}} \mathrm{FEM}\right)$ approximately equal to the value $p_{\mathrm{y}}$ determined experimentally $\left(p_{\mathrm{y}} \mathrm{FEM} / 3=p_{\mathrm{y}} / 3\right.$ $\cong \sigma_{\mathrm{y}}$ ). In these conditions (extended plastic flow under the indenter), the empirical relation used in the present work to correlate $p_{\mathrm{y}}$ and yield stress, is analogous to one of the main 


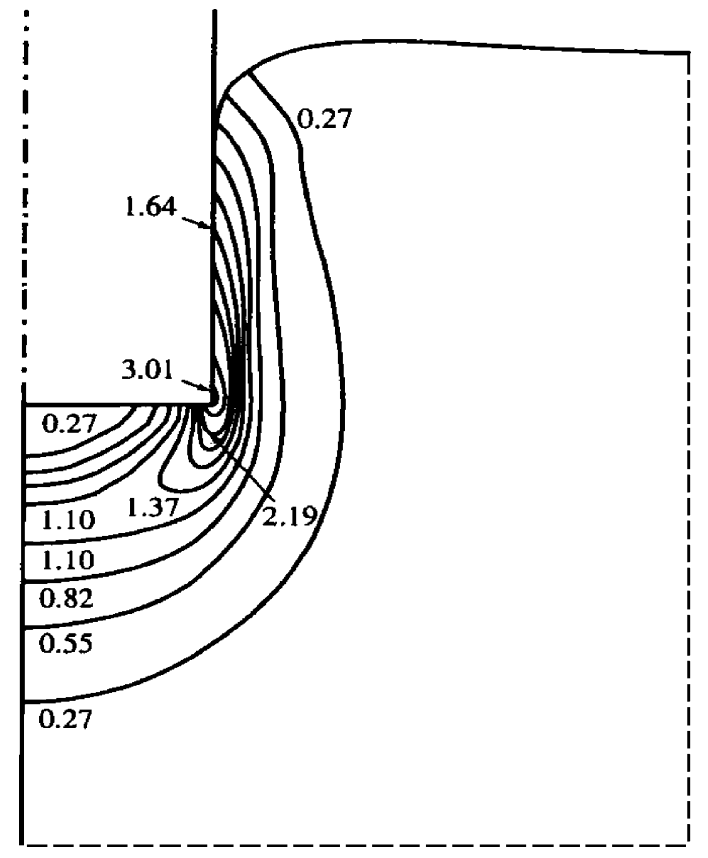

Fig. 12. Von Mises equivalent strain pattern following a 1-mm depth penetration estimated by FE analysis.

findings of the Shield and Eason work carried out by means of slip-line theory [34,35], i.e. the mean contact pressure is about six times the shear strength and as a consequence of Tresca plasticity criterion about three times the tensile yield stress.

Therefore, one can conclude that the $p_{\mathrm{y}}$ pressure determined experimentally is related to an extended plastic flow under the indenter and for the strain hardening materials to the initiation of the material pile up. The deformed shape and material pile up around the imprint are qualitatively similar to the experimental ones.

\section{Conclusions}

Considering the tests employing sharp punches, the flat-ended cylindrical indentation is characterised by a constant punch-sample contact area; thus, elastic modulus and yield stress can be directly determined from pressure-penetration curves by applying simple analytical relationships.

The experimental apparatus (FIMEC), developed by us, has been described. It has been used for investigating several metallic materials, including pure metals, steels and refractory alloys.

The yield stress can be determined by means of a characteristic pressure $\left(p_{\mathrm{y}}\right)$ clearly evidenced in the curves obtained for all tested materials: this pressure, as confirmed also by a FE analysis, is related to the full plasticity of the contact area and the beginning of material pile-up around the imprint.
The yield stress values experimentally determined exhibit relative deviations from those obtained by standard tensile tests not exceeding 9\%; a value similar to the scattering of data in tensile tests on the same material. The deviation for the elasticity modulus is about $3 \%$.

Furthermore, the variation of the slope $\Delta p / \Delta h$ in the third plastic stage of pressure-penetration curves, when tests are performed at different temperatures, can be exploited to estimate the DBTT of those metals which show such phenomenon. In particular, the DBTT data determined experimentally are very close to those obtained in Charpy tests with sub-sized KLST samples.

In this paper, the main theoretical aspects of flat-ended cylindrical indentation have been examined and a FE analysis has been performed to provide basic understanding of the plasticity mechanisms governing the indentation and their effect on pressure-penetration curve.

$\begin{array}{ll}\text { List of symbols } \\ a & \text { indenter radius } \\ A & \text { indentation contact area } \\ E & \text { sample elasticity (Young's) modulus } \\ E_{\mathrm{eq}} & \text { indentation equivalent elasticity modulus } \\ E_{\mathrm{i}} & \text { indenter elasticity modulus } \\ f & \text { friction coefficient } \\ h & \text { penetration depth } \\ k & \text { shear strength } \\ n & \text { hardening rate in Holloman's relationship } \\ p & \text { indentation pressure } \\ p_{\mathrm{L}} & \text { linear limit of the experimental } \\ & \text { pressure-penetration curve } \\ p_{\mathrm{m}} & \text { indentation mean pressure } \\ p_{\mathrm{y}} & \text { limit of the first plastic stage of the } \\ & \text { experimental } \\ p_{\mathrm{S}} & \text { pressure-penetration curve } \\ & \text { saturation limit of the experimental } \\ p-h \text { curve } & \text { pressure-penetration curve } \\ P & \text { mean contact pressure-penetration } \\ (r, \theta, z) & \text { indentation load } \\ S & \text { local cylindrical coordinate system } \\ u_{z} & \text { contact stiffness } \\ \sigma_{r} & \text { displacement in } z \text { direction } \\ \sigma_{\theta} & \text { normal stress in } r \text { direction } \\ \sigma_{z} & \text { normal stress in } \theta \text { direction } \\ \tau_{r z} & \text { normal stress in } z \text { direction } \\ v & \text { shear stress in the } r z \text { plane } \\ v_{\mathrm{i}} & \text { sample Poisson's coefficient } \\ \sigma_{\mathrm{y}} & \text { indenter Poisson's coefficient } \\ & \text { yield stress } \\ & \end{array}$

\section{References}

[1] O.D. Sherby, P.E. Armstrong, Metall. Trans. 2 (1971) 3479.

[2] T.R.G. Kutty, C. Ganguly, D.H. Sastry, Scripta Mater. 34 (1996) 1833. 
[3] A.G. Evans, E.A. Charles, J. Am. Ceram. Soc. 59 (1976) 371.

[4] G.R. Anstis, P. Chantikul, D.B. Marshall, B.R. Lawn, J. Am. Ceram. Soc. 64 (1981) 533.

[5] Y.Y. Lim, M.M. Chaudhri, J. Mater. Res. 14 (1999) 2314.

[6] W.S. Liggett, S.R. Low, D.J. Pitchure, J.F. Song, in: Proceedings of the XV IMEKO, Osaka, June 1999, p. 914.

[7] W. Yu, J.P. Blanchard, J. Mater. Res. 11 (1996) 2358.

[8] A.K. Bhattacharya, W.D. Nix, Int. J. Solids Struct. 24 (1988) 1287.

[9] A.E. Giannakopoulos, P.L. Larsson, R. Vestergaard, Int. J. Solids Struct. 31 (1994) 2679.

[10] E.C. Yu, J.C.M. Li, Philos. Mag. 36 (1977) 911.

[11] E.C. Yu, J.C.M. Li, J. Mater. Sci. 12 (1977) 2200.

[12] R. Mouginot, D. Maugins, J. Mater. Sci. 20 (1985) 4354.

[13] H.Y. Yu, M.A. Imam, B.B. Rath, J. Mater. Sci. 20 (1985) 636.

[14] P. Gondi, R. Montanari, A. Sili, J. Nucl. Mater. 212-215 (1994) 1688.

[15] J.I. Eldridge, D. Zhu, R.A. Miller, J. Am. Ceram. Soc. 84 (2001) 2737.

[16] P. Gondi, A. Donato, R. Montanari, A. Sili, J. Nucl. Mater. 233-237 (1996) 1557.

[17] P. Gondi, R. Montanari, A. Sili, S. Foglietta, A. Donato, G. Filacchioni, Fusion Technol. (1996) 1607.

[18] B. Riccardi, R. Montanari, L.F. Moreschi, A. Sili, S. Storai, Fusion Eng. Des. 58-59 (2001) 755.

[19] A. Donato, P. Gondi, R. Montanari, F. Moreschi, A. Sili, S. Storai, J. Nucl. Mater. 258-263 (1998) 446.

[20] S. Missori, R. Montanari, A. Sili, La Metallurgia Italiana 3 (2001) 35 .

[21] G. Filacchioni, R. Montanari, M.E. Tata, L. Pilloni, J. Nucl. Mater. 307-311 (2002) 1563.

[22] H. Hertz, J. Reine Angew. Math. 92 (1882) 156.

[23] V. Cerruti, Memorie di Fisica Matematica, Accademia dei Lincei, Roma, 1882.
[24] J. Boussinesq, Application des Potentiels a l'Etude de l'Equilibre et du Mouvement de Solides Elastiques-Gautier-Villar, Paris, 1885.

[25] A.E.H. Love, A Treatise on the Mathematical Theory of Elasticity, fourth ed. Dover Publications, New York, 1944, p. 515.

[26] I.N. Sneddon, Int. J. Eng. Sci. 3 (1965) 47.

[27] S. Timoshenko, J.N. Godier, Theory of Elasticity, third ed., Mc Graw Hill, New York, 1951, p. 408.

[28] I.N. Sneddon, Fourier Transforms, Mc-Graw-Hill, Singapore, 1970.

[29] D. Lorenz, Untersuchungen zur homogenen Versetzungnukleation mittels Nanoindentierung, Dr.rer.nat dissertation. Martin Luther Universitaet, Halle-Wittemberg, 2001, p. 5.

[30] I.S. Gradshteyn, I.M. Ryzhik, Table of Integrals, Series and Product, Academic Press, London, 1994, p. 154.

[31] R.F. Bishop, R. Hill, N.F. Mott, Proc. Phys. Soc. London 57 (1945) 147.

[32] K.L. Johnson, Contact Mechanics, Cambridge University Press, 1985, p. 153.

[33] K. Lange, Handbook of Metal Forming, Mc Graw Hill, NewYork, 1980, p. 12.

[34] R.T. Shield, Proc. R. Soc. A 233 (1955) 267.

[35] G. Eason, R.T. Shield, Zeitschrift für Angewandte Mathematik und Physik 11 (1960) 33

[36] ABAQUS/Standard Vers.5.8 Users' Manual, Hibbit Karlson and Sorensen Inc., Pawtucket, RI, 1998.

[37] ABAQUS/Explicit Users' Manual, Hibbit Karlson and Sorensen Inc., Pawtucket, RI, 1998.

[38] M.M. Chaudhri, Acta Mater. 46 (1998) 3042.

[39] B.W. Christ, Effect of Specimen Preparation, Setup and Test Procedures on Test Results, Metals Handbook, ninth ed., vol. 8, p. 32.

[40] W.C. Oliver, G.M. Pharr, J. Mater. Res. 7 (1998) 1564.

[41] D. Tabor, Proc. R. Soc. A 192 (1948) 247.

[42] W. Alexander, J. Shackelford, CRC Material Science and Engineering Handbook, third ed., CRC Press Inc., Boca Ratom, FL, 1997, p. 508 